\title{
Tide-induced head fluctuations in coastal aquifers of variable thickness
}

\author{
Julián Eduardo Cuello ${ }^{1,2}$ @ | Luis Guarracino ${ }^{1,2,3}$
}

\author{
${ }^{1}$ Facultad de Ciencias Astronómicas y \\ Geofísicas (FCAGLP), Universidad Nacional de \\ La Plata, La Plata, Argentina \\ ${ }^{2}$ Consejo Nacional de Investigaciones \\ Científicas (CONICET), Buenos Aires, \\ Argentina \\ ${ }^{3}$ Facultad de Ciencias Naturales y Museo \\ (FCNyM), Universidad Nacional de La Plata, La \\ Plata, Argentina \\ Correspondence \\ Julián Eduardo Cuello, Facultad de Ciencias \\ Astronómicas y Geofísicas (FCAGLP), \\ Universidad Nacional de La Plata, La Plata, \\ Argentina. \\ Email: julianecuello@fcaglp.unlp.edu.ar
}

\begin{abstract}
In this work, a new analytical solution to describe tide-induced head fluctuations in aquifers of variable thickness is presented. The proposed model assumes a finite and confined aquifer with a thickness that increases or decreases quadratically with the distance to the coast. A closed-form analytical solution is obtained by solving a boundary-value problem with both a separation of variables method and a change of variables method. This solution is a generalization of the solution obtained by Cuello et al., Hydrogeological Journal, 2017, 25, 1509-1515. The analytical solution is expressed in terms of the wedging parameter, a parameter that depends on the length and thicknesses at the coast and at the inland edge of the aquifer. Positive values of the wedging parameter describe aquifers with increasing thickness towards land and negative values describe aquifers with a decreasing thickness in the inland direction. The comparison of the new solution and the solution for a finite aquifer with constant thickness indicates that the sign of the wedging parameter enhances or decreases the amplitude of the tide-induced signal. However, the differences in time-lag between both solutions are negligible near the coast. The slope factor, which quantifies the inconsistencies between aquifer diffusivities estimated from attenuation and time-lag data, is computed and analysed. Near the coast, slope factor values greater than one are obtained for negative wedging parameters while slope factor values less than one are obtained for positive wedging parameters. The analysis of the new solution also indicates that more reliable estimates of the hydraulic diffusivity can be obtained from time-lag data.
\end{abstract}

\section{KEYWORDS}

amplitude attenuation, aquifers of variable thickness, hydraulic diffusivity, slope factor, tideinduced head fluctuations, time-lag

\section{1 | INTRODUCTION}

Most coastal aquifers are affected by ocean tides. The study of the hydraulic interaction between groundwater and seawater induced by tides provides the basis for a hydrogeophysical method for estimating hydraulic parameters. The tide-induced method consists of estimating the hydraulic diffusivity from the analysis of groundwater fluctuations induced by sea tides measured in wells located near the coastline.
This method is non-invasive, so it can be applied in contaminated areas as well as in coastal aquifers affected by seawater intrusion (Chattopadhyay, Vedanti, \& Singh, 2015; Jha, Kamii, \& Chikamori, 2003). Several analytical solutions which describe tide induced head fluctuations have been derived in the last decades (Li \& Jiao, 2003a). Ferris (1951) and Jacob (1950) were the first researchers to obtain the solution for a homogeneous confined aquifer that extends landward infinitely. This simple solution has been widely used for estimating the hydraulic 
diffusivity in coastal aquifers (Carr \& van der Kamp, 1969; Rotzoll, ElKadi, \& Gingerich, 2008; Rotzoll, Gingerich, \& El-Kadi, 2013; Zhou et al., 2015). However, the assumption of homogeneity of the aquifer could have significant discrepancy from real aquifers, which may show spatial variations of the hydraulic properties or complex geometries of the aquifer layer (Li \& Jiao, 2003b; Trefry \& Bekele, 2004).

It is important to remark that even though seawater intrusion is a major problem in most coastal aquifers (Werner et al., 2013), the present study focuses on the tidal-induced hydraulic interaction between the sea and the coastal aquifers. Fluid density effects have a little influence on tidal propagation (Slooten, Carrera, Castro, \& FernandezGarcia, 2010), so virtually all analytical solutions to describe this phenomenon are obtained under the assumption of constant density flow.

Analytical solutions of tide-induced head fluctuations for aquifers with heterogeneous hydraulic properties have been derived by several authors (Guarracino \& Monachesi, 2014; Guo, Jiao, \& Li, 2010; Monachesi \& Guarracino, 2011; Trefry, 1999; Wang, Zhan, \& Tang, 2015). Examples of complex geometries are difficult to find in the literature because most analytical solutions assume that the aquifer has a constant thickness. This hypothesis is not always valid because non-uniformity in aquifer thickness is commonly reported (Leray, Dreuzy, Bour, Labasque, \& Aquilina, 2012; Masterson et al., 2015; Okuyama et al., 2002; Refsgaard, 1997; Rotzoll et al., 2013). The deposition pattern of the sediments, the pre-depositional topography and the post-depositional erosion can lead to a widening or a thinning of the aquifer in the coastal zone (Rotzoll et al., 2013; Trapp, 1992). Zamrsky, Oude Essink, and Bierkens (2018) estimated the thickness of unconsolidated aquifers along the global coastline using available datasets. They assumed that this type of aquifers has an increasing thickness towards the coast and constitutes around $25 \%$ of the coastal ribbon of the world. Based on lithology and topographic information, they estimated the aquifer thickness by fitting both linear and quadratic trends. Hantush proposed linear and exponential laws to describe variations of the aquifer thickness (Hantush, 1962a,b,c). To the authors' knowledge, Cuello, Guarracino, and Monachesi (2017) derived the first analytical solution for tide-induced fluctuations that considers spatial variations in the aquifer thickness. This solution also provides a simple criterion to predict a possible thickness variation of the aquifer based on the computation of the slope factor introduced by Trefry and Bekele (2004).

The purpose of this study is to derive an analytical solution to describe tide induced head fluctuations in aquifers with quadratically increasing or decreasing thickness in the inland direction. The solution is obtained by solving a boundary-value problem combining the separation of variables and change of variables methods. This solution is a generalization of the solution obtained by Cuello et al. (2017) for an aquifer whose thickness decreases with the distance to the coast. The effect of widening or thinning of the aquifer on the induced head fluctuations is analysed in terms of a wedging parameter. Induced signals for aquifers with different wedging parameter values are compared with the induced heads of an aquifer with constant thickness. This comparison shows that induced head fluctuations are highly sensitive to thickness variations. It is also noted that the amplitude attenuation is strongly dependent on the wedging parameter while the time-lag is less sensitive, in particular near the coastline. This result suggests that time-lag data is more reliable to estimate the hydraulic diffusivity than amplitude data, when no prior information about the variation of the thickness is available. The slope factor, which quantifies the heterogeneity degree of the aquifer by comparing the hydraulic diffusivities estimated from the amplitude and time-lag, is also calculated and analysed for an increasing and decreasing thickness of the aquifer. The value of the slope factor near the coast can be used as an indicator of a thickness variation of the aquifer.

\section{2 | MATHEMATICAL MODEL AND ANALYTICAL SOLUTION}

In this section, the governing differential equation for groundwater flow and the analytical solution are presented. To obtain a closedform solution, a quadratic law is selected to describe the thickness variation. Let us consider a coastal confined aquifer of length $L$ whose thickness $b(x)(0<x<L)$ varies with the distance according to the following law:

$$
b(x)=b_{0}(1+\alpha x)^{2}
$$

where $\alpha$ is the wedging parameter defined as:

$$
\alpha=\frac{1}{L}\left(\sqrt{\frac{b_{L}}{b_{0}}}-1\right)
$$

being $b_{L}$ and $b_{0}$ the aquifer thicknesses at the coast $(x=0)$ and at the inland edge $(x=L)$, respectively. This parameter allows to describe aquifers with thicknesses that increase $(\alpha>0)$ or decrease $(\alpha<0)$ with the distance to the coast, as shown in Figure 1. Equation (1) represents an intermediate approach between linear and exponential variations proposed by Hantush (1962a,b,c). For the mathematical description of the problem, let the $x$-axis be perpendicular to the coastline, horizontal and positive landward, with the origin at the coast. The datum of the induced head fluctuations $(z=0)$ is the mean water level of the sea.

In order to derive an analytical solution, the following assumptions are made: (a) the flow in the confined aquifer is horizontal and obeys Darcy's law; (b) the effect of density variations on water flow is neglected and (c) the hydraulic conductivity $K_{s}\left(L T^{-1}\right)$ and the specific storativity $S_{s}\left(L^{-1}\right)$ are constant. According to these assumptions, tideinduced head fluctuations are described by the following equation (Bear, 1972):

$$
\frac{\partial}{\partial x}\left(T(x) \frac{\partial h}{\partial x}\right)=S(x) \frac{\partial h}{\partial t}
$$



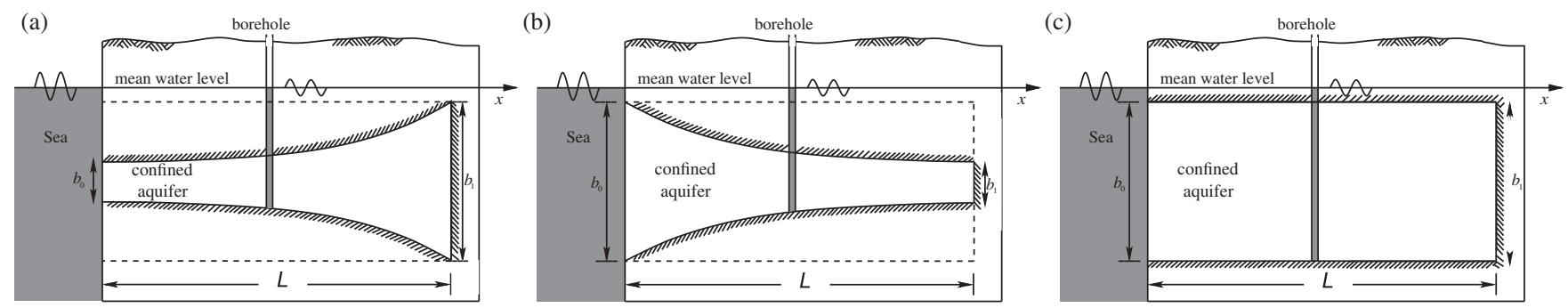

FIGURE 1 Schematic profile of aquifers with variable thicknesses. Panel (a) shows an aquifer with an increasing thickness ( $\alpha>0)$, panel (b) shows an aquifer with a decreasing thickness $(\alpha<0)$ and panel (c) shows an aquifer with a constant thickness $(\alpha=0)$

where $h(x, t)$ is the groundwater head $(L), T(x)=K_{s} b(x)$ the transmissivity $\left(L^{2} T^{-1}\right)$ and $S(x)=S_{s} b(x)$ the storativity $(-)$. Assuming that the sea tide can be described by a sinusoidal function (Kosuth et al., 2009), the boundary condition at the sea-aquifer interface $(x=0)$ can be expressed as:

$$
h(0, t)=A \cos (\omega t)
$$

where $A$ is the tidal amplitude $(\mathrm{L})$ and $\omega$ the tidal angular frequency $\left(\mathrm{T}^{-1}\right)$. It is important to remark that since the differential problem is linear, solutions for multiple-constituents tides can be obtained using the superposition principle. At the inland edge of the aquifer $(x=L)$ the following no-flow boundary condition is imposed:

$$
\lim _{x \rightarrow L}\left(T(x) \frac{\partial h}{\partial x}\right)=0
$$

This no-flow condition can also be used for aquifers which are not confined at $x=L$ since the amplitude of induced heads are highly attenuated near the coast and the boundary effects can be considered negligible.

The exact analytical solution of Equations (3)-(5) can be obtained by rewriting the differential problem in complex form and solving the equation using the separation of variables method and change of variables method. The exact analytical solution is as follows (see Appendix for derivation):

$$
\begin{aligned}
& h(x, t)= \frac{A p}{(1+\alpha x)}\left[e^{a x} \cos (\omega t+a x)-e^{a(2 L-x)}\left(\cos (\omega t+a(2 L-x)) s_{1}\right)\right. \\
&\left.-\sin (\omega t+a(2 L-x)) s_{2}\right]-\frac{A q}{(1+\alpha x)} \\
& \quad \times\left[e^{a x} \sin (\omega t+a x)-e^{a(2 L-x)}\left(\cos (\omega t+a(2 L-x)) s_{2}+\sin (\omega t+a(2 L-x)) s_{1}\right)\right]
\end{aligned}
$$

where:

$$
p=\frac{1-e^{2 a L}\left(\cos (2 a L) s_{1}+\sin (2 a L) s_{2}\right)}{\left[1-e^{2 a L}\left(\cos (2 a L) s_{1}+\sin (2 a L) s_{2}\right)\right]^{2}+e^{4 a L}\left(\cos (2 a L) s_{2}+\sin (2 a L) s_{1}\right)^{2}}
$$

$q=\frac{e^{2 a L}\left(\sin (2 a L) s_{1}+\cos (2 a L) s_{2}\right)}{\left[1-e^{2 a L}\left(\cos (2 a L) s_{1}+\sin (2 a L) s_{2}\right)\right]^{2}+e^{4 a L}\left(\cos (2 a L) s_{2}+\sin (2 a L) s_{1}\right)^{2}}$

and

$$
\begin{gathered}
s_{1}=\frac{\alpha^{2}-2 a^{2}(1+\alpha \mathrm{L})^{2}}{[\alpha+a(1+\alpha \mathrm{L})]^{2}+a^{2}(1+\alpha \mathrm{L})^{2}} \\
s_{2}=\frac{-2 \alpha a(1+\alpha \mathrm{L})}{[\alpha+a(1+\alpha \mathrm{L})]^{2}+a^{2}(1+\alpha \mathrm{L})^{2}} \\
a=\sqrt{\frac{\omega S_{s}}{2 K s}}
\end{gathered}
$$

Note that the solution (6) depends on the following independent geometrical and physical variables: (a) boundary signal amplitude and frequency $(A, \omega)$; (b) aquifer's hydraulic conductivity and specific storativity $\left(K_{s}, S_{s}\right)$ and (c) the wedging parameter $\alpha$, which defines the geometry of the aquifer $\left(b_{0}, b_{L}, L\right)$.

The proposed analytical solution is a generalization of the solution obtained by Cuello et al. (2017). Note that for $b_{L}=0(\alpha=-1 / L)$ the thickness of the aquifer defined by Equation (1) becomes $b(x)=b_{0}(1$ $-x / L)^{2}$, which is the same expression proposed by these authors for an aquifer thickness that decreases to zero with the distance to the coast.

The solution for a box-shaped aquifer (finite aquifer with constant thickness represented in Figure $1 c$ is obtained by assuming $b_{L}=b_{0}(\alpha=0)$ :

$$
\begin{aligned}
& h(x, t)=A p\left[e^{-a x} \cos (\omega t-a x)+e^{-a(2 L-x)} \cos (\omega t-a(2 L-x))\right] \\
&-A q\left[e^{-a x} \sin (\omega t-a x)-e^{-a(2 L-x)} \sin (\omega t-a(2 L-x))\right]
\end{aligned}
$$

where:

$$
p=\frac{1+e^{-2 a L} \cos (2 a L)}{1+e^{-2 a L} \cos (2 a L)+e^{-4 a L}}
$$




$$
q=\frac{e^{-2 a L} \sin (2 a L)}{1+e^{-2 a L} \cos (2 a L)+e^{-4 a L}}
$$

In the next section, Equation (12) will be used to analyse the effect of thickness variations on induced heads.

Finally, if $L$ tends to infinity ( $\alpha$ tends to zero), Equation (6) is reduced to:

$$
h(x, t)=A e^{-a x} \cos (\omega t-a x)
$$

which is the solution obtained by Jacob (1950).

\section{3 | RESULTS AND DISCUSSION}

In order to explore the effect of thickness variations on tide induced head fluctuations, the predicted responses of the proposed model (6) and the head fluctuations in a box-shaped aquifer (15) are compared. To perform this comparison the following aquifer parameters are used: $L=100 \mathrm{~m}, \alpha=10^{-2} \mathrm{~m}^{-1}\left(b_{L}=4 b_{0}\right), \alpha=-510^{-3} \mathrm{~m}^{-1}\left(b_{L}=b_{0} / 4\right)$ and $\alpha=0\left(b_{L}=b_{0}\right)$. The geometry of these aquifers is schematized in Figure 1. Note that the aquifers in Figure 1a,b are spectral images from each other. The assumed hydraulic properties are $K_{s}=50 \mathrm{md}^{-1}$ and $S_{s}=310^{-3} \mathrm{~m}^{-1}$, then the hydraulic diffusivity can be computed as $D=K_{s} / S_{s}=1.6710^{4} \mathrm{~m}^{2} \mathrm{~d}^{-1}$. The sea tide is assumed to be semidiurnal (period of $12.4 \mathrm{hr}$ ) with amplitude $A=1 \mathrm{~m}$.

Figure 2 shows time variations of the tide-induced heads for the three aquifers near the coast $(x=10 \mathrm{~m})$, at the midpoint $(x=50 \mathrm{~m})$ and near the inland edge $(x=90 \mathrm{~m})$. It can be noticed that in the three panels an increasing thickness (positive wedging parameter) produces smaller amplitudes of induced heads compared with the box-shaped aquifer solution, while a decreasing thickness (negative wedging parameter) produces larger amplitudes. Also note that time-lag differences between the three models can be observed at $x=50 \mathrm{~m}$ and $x=90 \mathrm{~m}$, where the aquifer with $\alpha=10^{-2} \mathrm{~m}^{-1}$ presents a larger timelag than the aquifer with $\alpha=-5 \times 10^{-3} \mathrm{~m}^{-1}$.

For a better understanding of the effect of thickness variations on induced heads, the maximum amplitude $h_{\max }$ and the time-lag $\tau_{\text {lag }}$ of the induced signals as a function of the distance $x$ are computed. These parameters have the following analytical expressions:

$$
\begin{gathered}
h_{\max }(x)=\sqrt{y_{r}^{2}(x)+y_{i}^{2}(x)} \\
\tau_{\text {lag }}(x)=-\frac{1}{\omega} \tan ^{-1}\left(\frac{y_{i}(x)}{y_{r}(x)}\right)
\end{gathered}
$$

where $y_{r}(x)$ and $y_{i}(x)$ are, respectively, the real and imaginary parts of the following complex equation, which is the solution of the differential problem (A4)-(A6) described in the Appendix:

$$
\begin{gathered}
y(x)=C_{1} \frac{e^{(1+i) a x}}{1+\alpha x}+C_{2} \frac{e^{-(1+i) a x}}{1+\alpha x} \\
C_{1}=\frac{A}{1+e^{2(1+i) a L}\left[\frac{\alpha-(1+i) a(1+\alpha L)}{\alpha+(1+i) a(1+\alpha L)}\right]} \\
C_{2}=-A \frac{e^{2(1+i) a L}\left[\frac{\alpha-(1+i) a(1+\alpha L)}{\alpha+(1+i) a(1+\alpha L)}\right]}{1+e^{2(1+i) a L}\left[\frac{\alpha-(1+i) a(1+\alpha L)}{\alpha+(1+i) a(1+\alpha L)}\right]}
\end{gathered}
$$

Figure 3 shows the maximum amplitudes and time-lags as functions of the distance to the coast $x$. It can be observed that an increasing thickness generates a reduced amplitude of the induced tide and a decreasing thickness produces an enhanced amplitude of the induced signal for the whole range of $x$ values. Thickness variations have virtually no effect on time-lags near the coast. For $x$ values greater than $40 \mathrm{~m}$ the time-lag is larger for the aquifer with a positive wedging parameter and smaller for the aquifer with a negative wedging parameter, comparing with the box-shaped aquifer time-lag.

In most practical applications of the tide-induced technique, the hydraulic diffusivity is obtained from both amplitude or time-lag data using the following expressions (Trefry \& Bekele, 2004):
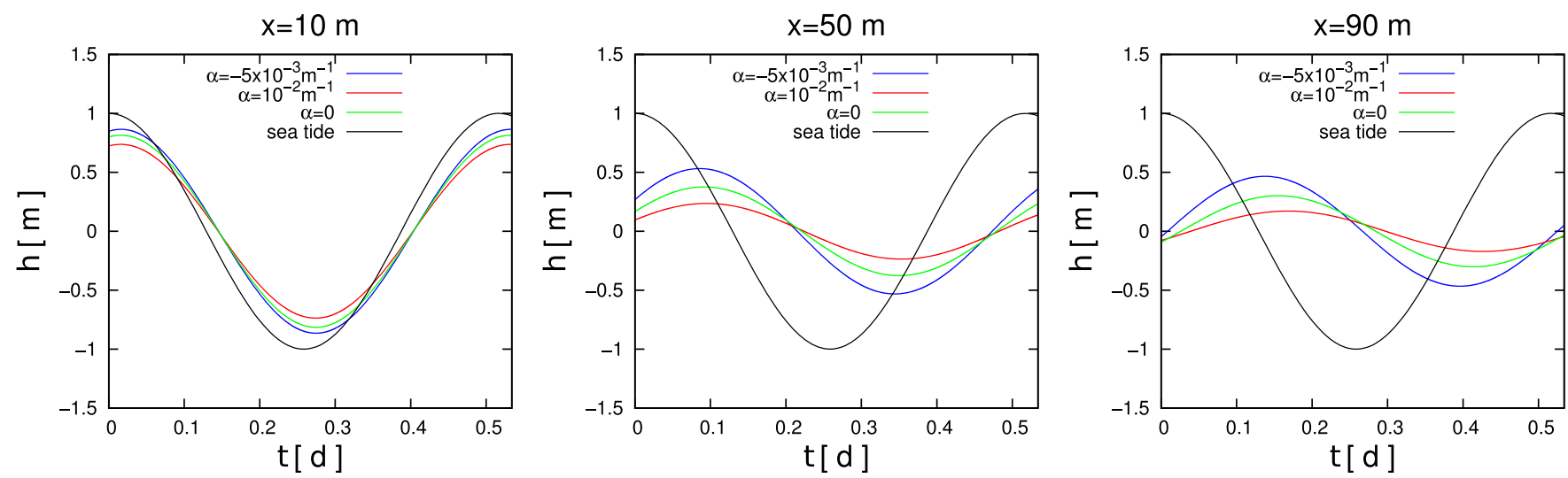

FIGURE 2 Time variations of the tide-induced head fluctuations estimated for three values of $\alpha$ at three different distances from the coastline. Sea tide is included in the figure 

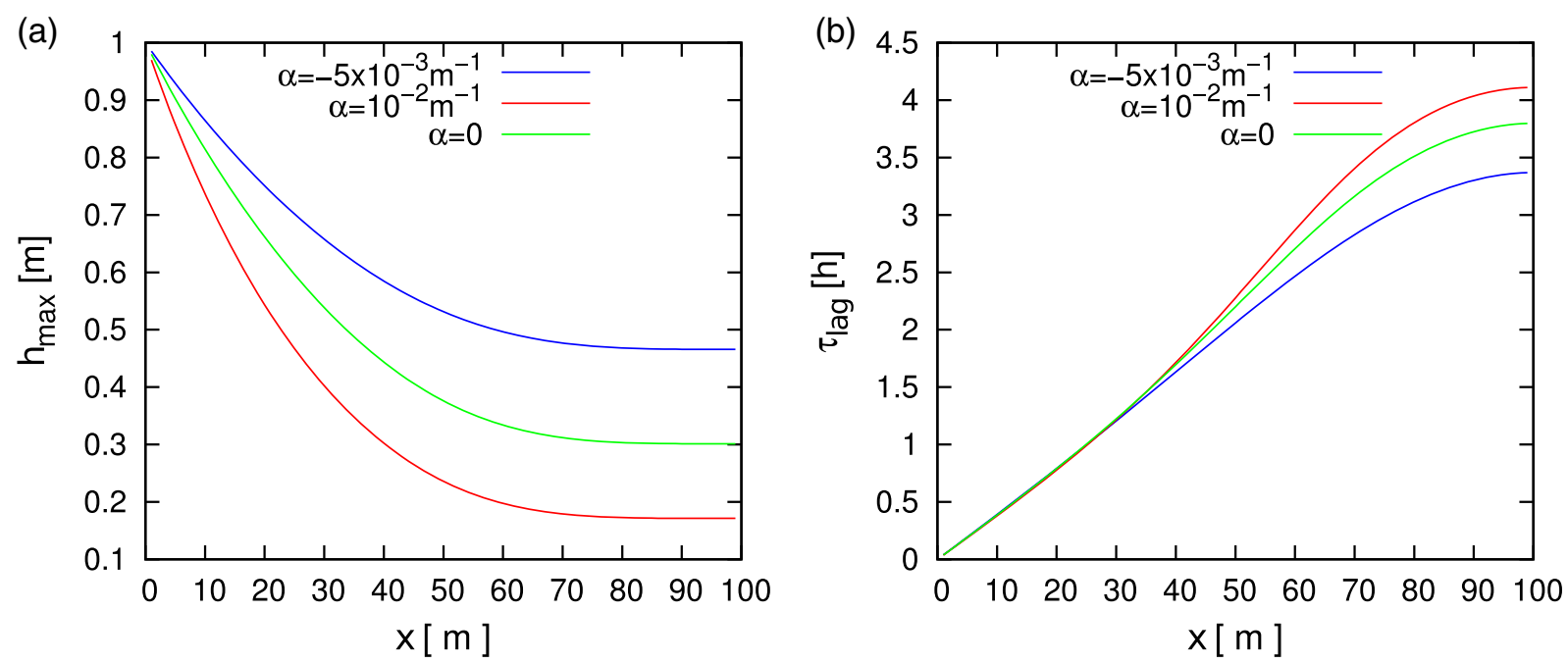

FIGURE 3 (a) Maximum amplitudes and (b) time-lags versus distance to the coastline for different aquifer wedging parameters

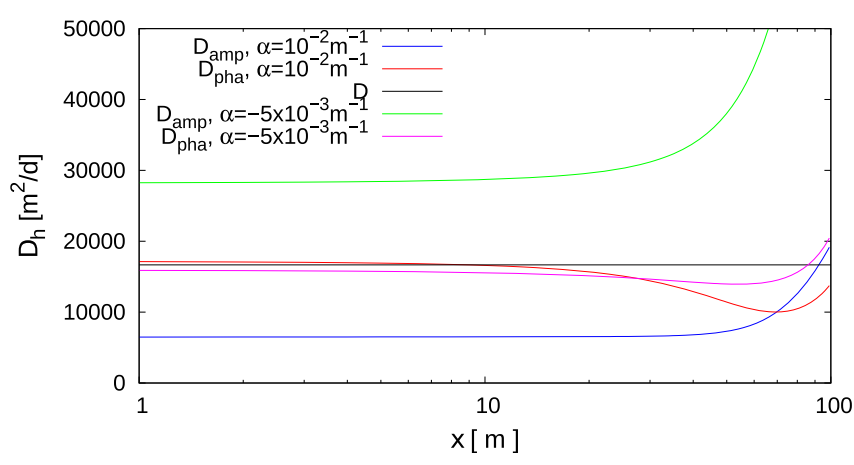

FIGURE 4 Hydraulic diffusivities obtained with time-lag and amplitude data and real hydraulic diffusivity

$$
\begin{aligned}
& D_{a m p}=\frac{x^{2} \omega}{2 \ln ^{2}\left[\frac{h_{\max }(x)}{A}\right]} \\
& D_{p h a}=\frac{x^{2}}{2 \omega \tau_{\text {lag }}^{2}(x)} .
\end{aligned}
$$

The above equations are obtained from Jacob's solution which assumes a homogeneous, infinity and constant thickness aquifer. Under these hypothesis, the aquifer hydraulic diffusivity can be obtained by using Equations (21) or (22), since $D=D_{a m p}=D_{\text {pha }}$. In order to test the validity of these equations in aquifers with variable thickness the amplitude $h_{\max }(x)$ and time-lag $\tau_{\operatorname{lag}}(x)$ are computed using Equations (16) and (17). Figure 4 shows the hydraulic diffusivities obtained with time-lag and amplitude data for the aquifers with positive and negative wedging parameter and the actual hydraulic diffusivity of the aquifer $D=K_{s} /$ $S_{s}=1.6710^{4} \mathrm{~m}^{2} \mathrm{~d}^{-1}$ versus the distance to the coast.

Note that the diffusivity values obtained from amplitude data significantly overestimate or underestimate the diffusivity $D$ for negative and positive values of the wedging parameter, respectively. However, near

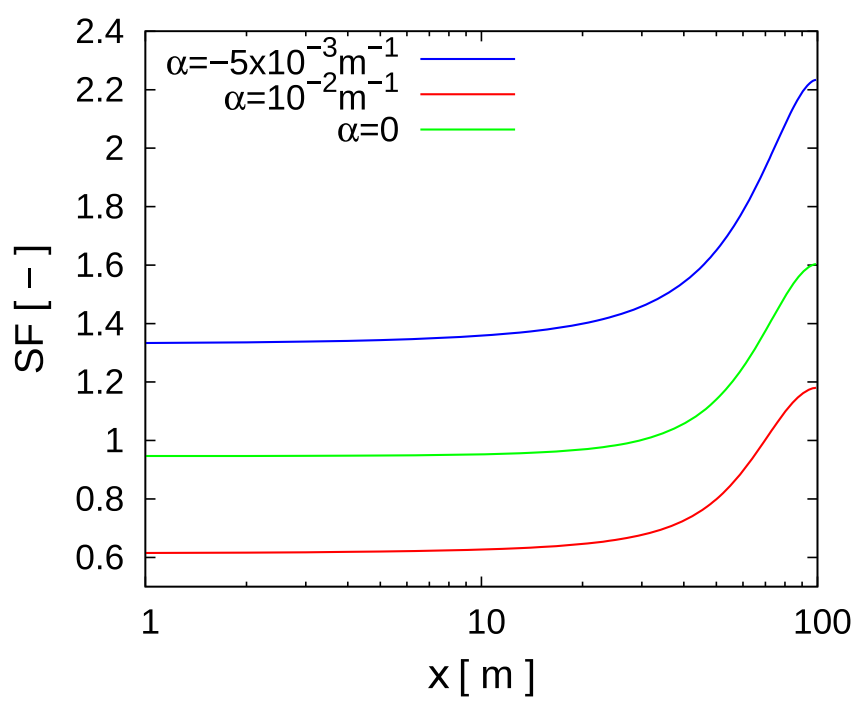

FIGURE 5 Slope factor versus distance to the coast for an aquifer with increasing thickness and an aquifer with decreasing thickness . Box-shaped slope factor is represented by a green line

the coast the diffusivity values obtained using time-lag data provide better estimates of the real diffusivity $D$ for both aquifers with positive and negative wedging parameters. This result is based on the fact that near to the coast the time-lag is not sensitive to thickness variations (Figure 3). It can be concluded that Equation (22) should be used to obtain a better estimate of the hydraulic diffusivity when no prior information about a possible variation in the thickness of the aquifer is available.

The slope factor SF, which quantifies the differences between $D_{a m p}$ and $D_{p h a}$ is expressed as follows (Trefry and Bekele (2004)):

$$
S F(x)=\sqrt{\frac{D_{a m p}}{D_{p h a}}} .
$$



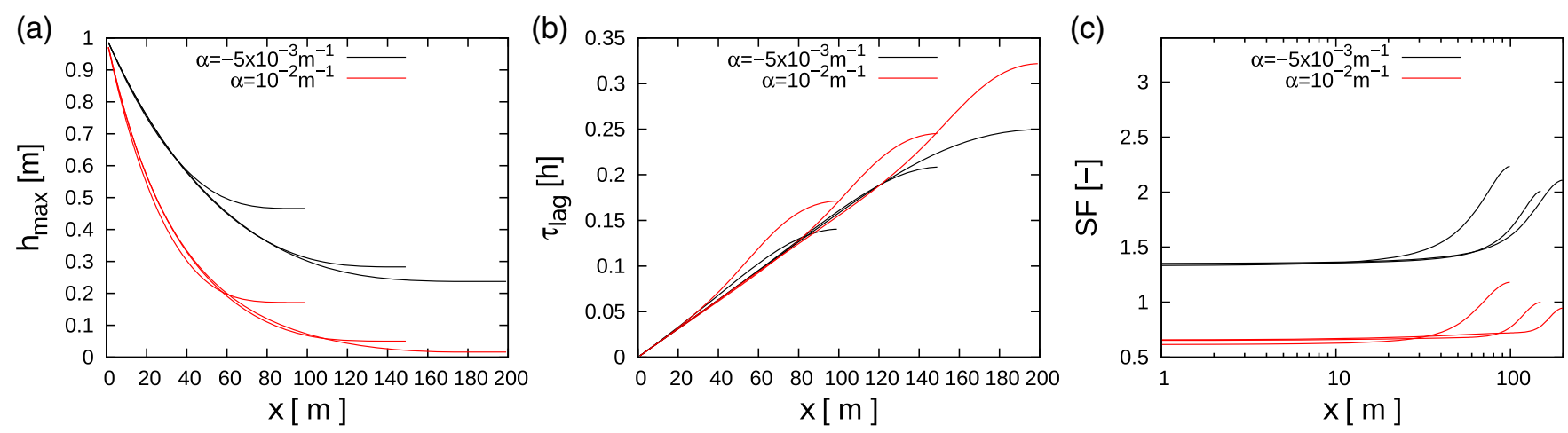

FIGURE 6 (a) Amplitude, (b) time lag and (c) slope factor versus distance to the coastline for different aquifer lengths $L=100 \mathrm{~m}, L=150 \mathrm{~m}$ and $L=200 \mathrm{~m}$

For a homogeneous and infinite aquifer the slope factor is constant and equal to unity. Other values of SF indicate inhomogeneities of the aquifer properties. Thus, the slope factor can be considered as an indicator of the heterogeneity degree of the aquifer. Figure 5 shows the slope factor as a function of the distance to the coast for the three aquifers described in this section. Near the coast, a decreasing thickness (negative wedging parameter) produces a slope factor greater than 1. Similar results are reported by Cuello et al. (2017) for an aquifer whose thickness decreases to zero. Conversely, an increasing thickness (positive wedging parameter) produces a slope factor smaller than 1 near the coast.

In the next example the effect of the length $L$ of the aquifer in the induced heads is analysed. Let us consider six different aquifers of length $L=100 \mathrm{~m}, L=150 \mathrm{~m}$ and $L=200 \mathrm{~m}$ with both positive and negative wedging parameters $\alpha=10^{-2} \mathrm{~m}^{-1}$ and $\alpha=-5 \times 10^{-3} \mathrm{~m}^{-1}$. Using Equations (16), (17) and (23), the amplitude, time-lag and slope factor as a function of the distance to the coast are computed for the six aquifers.

Figure 6a shows the effect of the aquifer length on the maximum amplitude of the induced head fluctuations. The effect of the aquifer length is significant near the inland edge and almost negligible near the coast. Similarly, time-lag differences are undetectable near the coast, but close to the inland edge of the aquifers the differences increase (Figure 6b). Finally, slope factors SF are shown in Figure 6c. As it is expected, the effect of the length on SF is significant far away from the coast. The analysis of this figure allows to conclude that a positive wedging parameter generates slope factors values less than unity and a negative wedging parameter generates slope factor values greater than one, regardless the length of the aquifer. However, close to the inland edge of the aquifer this criterion is no longer valid.

\section{$4 \mid$ CONCLUSIONS}

In this study, a new analytical solution to describe tide-induced head fluctuations in confined coastal aquifers with non-uniform thickness is derived. The aquifer is assumed to be finite with a thickness that follows a quadratic law, which allows to describe aquifers with continuously increasing or decreasing thicknesses. For this aquifer geometry, a closed-form analytical solution is obtained in terms of a wedging parameter. Positive values of this parameter describe aquifers with increasing thickness and negative values describe aquifers with decreasing thickness. This solution is a generalization of the solution obtained by Cuello et al. (2017) for an aquifer with a decreasing thickness that tends to zero.

The analysis of the analytical solution shows that the effect of thickness variations is significant on the induced amplitude but negligible on time-lag near the coast. Furthermore, the slope factor, which measures the degree of heterogenity, allows to infer the type of thickness variation. Slope factor values greater than 1 near the coast indicate an increasing thickness and values less than 1 indicate a decreasing thickness. Another important finding of this study is that the hydraulic diffusivity value obtained from time-lag data using the standard procedure (Equation 22) is more reliable than the value estimated from amplitude data (Equation 21). This result has practical implications for the estimate of hydraulic diffusivity using the tideinduced method when no information about the aquifer thickness variation is available.

\section{DATA AVAILABILITY STATEMENT}

Data sharing is not applicable to this article as no new data were created or analysed in this study.

\section{ORCID}

Julián Eduardo Cuello (iD https://orcid.org/0000-0002-3429-5375

\section{REFERENCES}

Bear, J. (1972). Dynamics of fluids in porous media, New York: Dover.

Carr, P. A., \& van der Kamp, G. (1969). Determining aquifer characteristics by the tidal methods. Water Resources Research, 5(5), 1023-1031.

Chattopadhyay, P. B., Vedanti, N., \& Singh, V. (2015). A conceptual numerical model to simulate aquifer parameters. Water Resources Manage, 29, 771-784.

Cuello, J. E., Guarracino, L., \& Monachesi, L. (2017). Groundwater response to tidal fluctuations in wedge-shaped confined aquifers. Hydrogeological Journal, 25, 1509-1515. https://doi.org/10.1007/ s10040-017-1579-z 
Ferris, J. G. (1951). Cyclic fluctuations of water level as a basis for determining aquifer transmissibility. International Association of Scientific Hydrology, Publication, 33, 148-155.

Guarracino, L., \& Monachesi, L. (2014). An analytical solution of tideinduced head fluctuations in an inhomogeneous coastal aquifer. Lecture Notes in Earth System Sciences, 475-479. https://doi.org/10. 1007/978-3-642-32408-6_105.

Guo, H., Jiao, J., \& Li, H. (2010). Groundwater response to tidal fluctuations in a two-zone aquifer. Journal of Hydrology, 381, 364-371.

Hantush, M. (1962a). Flow of ground water in sands of non-uniform thickness. Part 1. Flow in a wedge-shaped aquifer. Journal of Geophysical Research, 67, 703-709.

Hantush, M. (1962b). Flow of ground water in sands of non-uniform thickness. Part 2. Approximate theory. Journal of Geophysical Research, 67, 711-720.

Hantush, M. (1962c). Flow of ground water in sands of non-uniform thickness. Part 3. Flow to Wells. Journal of Geophysical Research, 67, 1527-1534.

Jacob, C. E. (1950). Flow of groundwater. In H. Rouse (Ed.), Engineering hydraulics. New York, NY: Wiley.

Jha, M. K., Kamii, Y., \& Chikamori, K. (2003). On the estimation of phreatic aquifer parameters by the tidal response technique. Water Resources Management, 17, 69-88.

Kosuth, P., Callède, J., Laraque, A., Filizola, N., Guyot, J., Seyler, P., ... Guimarães, V. (2009). Sea-tide effects on flows in the lower reaches of the amazon river. Hydrological Processes: An International Journal, 23 (22), 3141-3150.

Leray, S., Dreuzy, J., Bour, O., Labasque, T., \& Aquilina, L. (2012). Contribution of age data to the characterization of complex aquifers. Journal of Hydrology, 464, 54-68.

Li, H., \& Jiao, J. J. (2003a). Review of analytical studies of tidal groundwater flow in coastal aquifer systems. In Proceedings of the international symposium on water resources and the urban environment (pp. 9-10). Wuhan, China: Water Resources and the Urban Environment.

$\mathrm{Li}, \mathrm{H}$., \& Jiao, J. J. (2003b). Influence of tide on the mean watertable in an unconfined, anisotropic, inhomogeneous coastal aquifer. Advances in Water Resources, 26, 9-16.

Masterson, J. P., Pope, J., Monti, J., Nardi, M., Finkelstein, J., \& McCoy, K. (2015). Hydrogeology and hydrologic conditions of the northern Atlantic coastal plain aquifer system from long Island, New York, to North Carolina. In U.S. geological survey scientific investigations report 2013-5133. Reston, Virginia: U.S. Geological Survey. https://doi.org/ 10.3133/sir20135133

Monachesi, L. B., \& Guarracino, L. (2011). Exact and approximate analytical solutions of groundwater response to tidal fluctuations in a theoretical inhomogeneous coastal confined aquifer. Hydrogeology Journal, 19, 1443-1449.

Okuyama, K., Fujiwara, M., Ohnishi, C., Watanabe, H., Tabira, K., \& Nishigaki, M. (2002). System for estimating coefficients of permeability in regional ground water survey. Journal of Japan Society of Civil Engineers, 701, 17-28.

Refsgaard, J. C. (1997). Parameterisation, calibration and validation of distributed hydrological models. Journal of Hydrology, 198, 69-97.

Rotzoll, K., El-Kadi, A., \& Gingerich, S. (2008). Analysis of an unconfined aquifer subject to asynchronous dual-tide propagation. Ground Water, 46, 239-250.

Rotzoll, K., Gingerich, S., \& El-Kadi, A. (2013). Estimating hydraulic properties from tidal attenuation in the northern Guam lens aquifer, territory of Guam, USA. Hydrogeology Journal, 21, 643-654.

Slooten, L. J., Carrera, J., Castro, E., \& Fernandez-Garcia, D. (2010). A sensitivity analysis of tide-induced head fluctuations in coastal aquifers. Journal of Hydrology, 393(3-4), 370-380.

Trapp, H. (1992). Hydrogeologic framework of the northern Atlantic coastal plain in parts of North Carolina, Virginia, Maryland, Delaware, New Jersey, and New York. US Geological Survey Professional Paper, 1404, 1-59.
Trefry, M. G. (1999). Periodic forcing in composite aquifers. Advances in Water Resources, 22, 645-656.

Trefry, M. G., \& Bekele, E. (2004). Structural characterization of an Island aquifer via tidal methods. Water Resources Research, 40, W01505. https://doi.org/10.1029/2003WR002003.

Wang, Q., Zhan, H., \& Tang, Z. (2015). Two-dimensional flow response to tidal fluctuation in a heterogeneous aquifer-aquitard system. Hydrological Processes, 29, 927-935.

Werner, A. D., Bakker, M., Post, V. E., Vandenbohede, A., Lu, C., AtaieAshtiani, B., ... Barry, D. A. (2013). Seawater intrusion processes, investigation and management: Recent advances and future challenges. Advances in Water Resources, 51, 3-26.

Zamrsky, D., Oude Essink, G. H., \& Bierkens, M. F. (2018). Estimating the thickness of unconsolidated coastal aquifers along the global coastline. Earth System Science Data, 10, 1591-1603.

Zhou, X., Song, C., Li, T., Chen, R., Zhang, H., Zhao, J., \& Cao, Q. (2015). Estimation of aquifer parameters using tide-induced groundwater level measurements in a coastal confined aquifer.

How to cite this article: Cuello JE, Guarracino L. Tide-induced head fluctuations in coastal aquifers of variable thickness. Hydrological Processes. 2020;34:4139-4146. https://doi.org/ 10.1002/hyp.13873

\section{APPENDIX A}

Let $H(x, t)$ be a complex function that satisfies the following boundary-value problem:

$$
\begin{gathered}
\frac{\partial}{\partial x}\left(T(x) \frac{\partial H}{\partial x}\right)=S(x) \frac{\partial H}{\partial t} \\
H(0, t)=A e^{i \omega t} \\
\lim _{x \rightarrow L} T(x) \frac{\partial H}{\partial x}=0
\end{gathered}
$$

Because $h(x, t)$ is the solution of (3)-(5), it follows that $h(x, t)=R e$ $(H(x, t))$, where Re denotes the real part of the complex expression. Substituting $H(x, t)=y(x) e^{i \omega t}$ into (A1)-(A3) yields:

$$
\begin{gathered}
(1+\alpha x) \frac{d^{2} y}{d x^{2}}+2 \alpha \frac{d y}{d x}-\frac{i \omega S_{s}}{K_{s}}(1+\alpha x) y=0 \\
y(0)=A \\
(1+\alpha x)^{2} \frac{d y}{d x}=0
\end{gathered}
$$

where $y(x)$ is a complex function and $a=\sqrt{\frac{\omega S_{s}}{2 K_{s}}}$ is the tidal propagation parameter.

In order to find the general solution of Equation (A4), the following change of variables is proposed: 


$$
v(x)=(1+\alpha x) y(x)
$$

(A7)

Replacing Equation (A7) in Equations (A4)-(A6) gives:

$$
\frac{d^{2} v}{d x^{2}}-2 i a^{2} v=0
$$

$$
v(0)=A
$$

$$
\lim _{x \rightarrow L}(1+\alpha x) \frac{d v}{d x}-\alpha v(x)=0
$$

By solving Equations (A8)-(A10), one obtains Equation (6) as the solution of the initial differential problem (3)-(5). 